\title{
Una revisión general de las estructuras metal-orgánicas (MOF) dentro de la química inorgánica
}

A general revision of metal-organic frameworks (MOF) within inorganic chemistry

\author{
M. H. Villegas-Fernández ${ }^{\mathrm{a}, 1}$ (D) M. Carpio-Granilloa,1D， E. Vargas-Hernández ${ }^{\mathrm{a}}$ (D), F. J. Zuno-Cruz ${ }^{\mathrm{a}}$ (D), \\ G. Sánchez-Cabrera iD a,* \\ a Área Académica de Química, Universidad Autónoma del Estado de Hidalgo, 42184, Pachuca, Hidalgo, México.
}

\begin{abstract}
Resumen
Las estructuras metal-orgánicas (MOF) son materiales porosos con arreglos espaciales ordenados de aspecto cristalino y están formados por ligantes orgánicos y iones o cúmulos metálicos. Este tipo de compuestos han sido estudiados durante algunas décadas, debido a los diversos campos de aplicación en donde se han involucrado. La principal razón para su estudio es la gran variedad de ligantes orgánicos y iones metálicos generalmente de transición que pueden ser utilizados para su síntesis, generando un gran número de variables en la geometría (2D o 3D) que controla el tamaño de poro de estos compuestos. Este artículo es una revisión general de las características significativas, los tipos de estructura y métodos sintéticos más importantes usados en la actualidad para MOF, además se mencionan algunas de sus aplicaciones más relevantes dentro de la química inorgánica.
\end{abstract}

\section{Palabras Clave:}

Estructuras metal-orgánicas, MOF, ligantes orgánicos, iones metálicos de transición.

\begin{abstract}
Metal-organic frameworks (MOFs) are porous materials with ordered spatial arrangements crystalline-looking and are made of organic linkers and metal ions or clusters. These types of compounds have been studied for some decades, due to the various fields of application in which they have been involved. The main reason for their study is the great variety of organic linkers and transition metal ions that can be used to be synthesized, generating many variables in geometry (2D or 3D) in order to control the pore size of these compounds. This article is a general revision of the significant characteristics, types of structure and the most important synthetic methods used today in MOF, and some relevant applications are mentioned within the inorganic chemistry field.
\end{abstract}

Keywords:

Metal-organic framework, MOF, organic linkers, transition metal ions.

\section{Introducción}

A lo largo de la historia, el hombre ha utilizado distintos materiales para llevar a cabo diversas actividades, las cuales han evolucionado conforme el conocimiento avanza y las necesidades cambian. Durante las últimas décadas el interés por el diseño y síntesis de materiales cristalinos ha crecido enormemente, lo cual ha llevado al surgimiento de nuevas especies químicas como son las estructuras metal-orgánicas (Metal-Organic Frameworks, MOF, por sus siglas en inglés).

Los MOF son redes de coordinación poliméricas cristalinas constituidos por dos grandes componentes: los ligantes orgánicos (que son moléculas orgánicas que tienen grupos funcionales adecuados para unirse químicamente a átomos metálicos) y los nodos inorgánicos, que son la parte metálica de las especies y que puede ser átomos únicos (iones) o cúmulos (agregados metálicos), y que forman redes bi- (2D) o tridimensionales (3D), como se esquematiza en la Figura 1.

Es importante mencionar que el incremento en el conocimiento sobre el diseño y la síntesis de manera racional a partir de bloques de construcción moleculares de los MOF condujo al surgimiento de una disciplina llamada química reticular (O’keeffe, 2018).

Debido a la relevancia que estas especies químicas han tenido en las ciencias químicas, en este artículo se describen algunos

\footnotetext{
*Autor para la correspondencia: gloriasa@uaeh.edu.mx

Correo electrónico: vi270173@uaeh.edu.mx (Milton Horacio Villegas-Fernández), ca170300@uaeh.edu.mx (Mariana Carpio-Granillo), va295893@uaeh.edu.mx (Erick VargasHernández), fjzuno@uaeh.edu.mx (Francisco Javier Zuno-Cruz), gloriasa@uaeh.edu.mx (Gloria Sánchez-Cabrera).

${ }^{1}$ M.H.V.F. y M. C. G. contribuyeron de igual forma a este trabajo. 
antecedentes e historia de su desarrollo, se presenta una sinopsis de algunas propiedades significativas de los MOF, se describen los tipos de estructuras que pueden presentar; además de que se mencionan generalidades de los métodos de síntesis descritos y se comentan algunas de las aplicaciones más relevantes de los MOF dentro de la química inorgánica. Esta revisión permitirá al lector entender de forma general las características estructurales y su relación entre la parte orgánica (ligantes) y la parte inorgánica (nodos) lo que ha permitido modular la formación de MOF afectando sus propiedades físicas y químicas y por lo tanto influir en el tipo de aplicaciones que podrían tener.

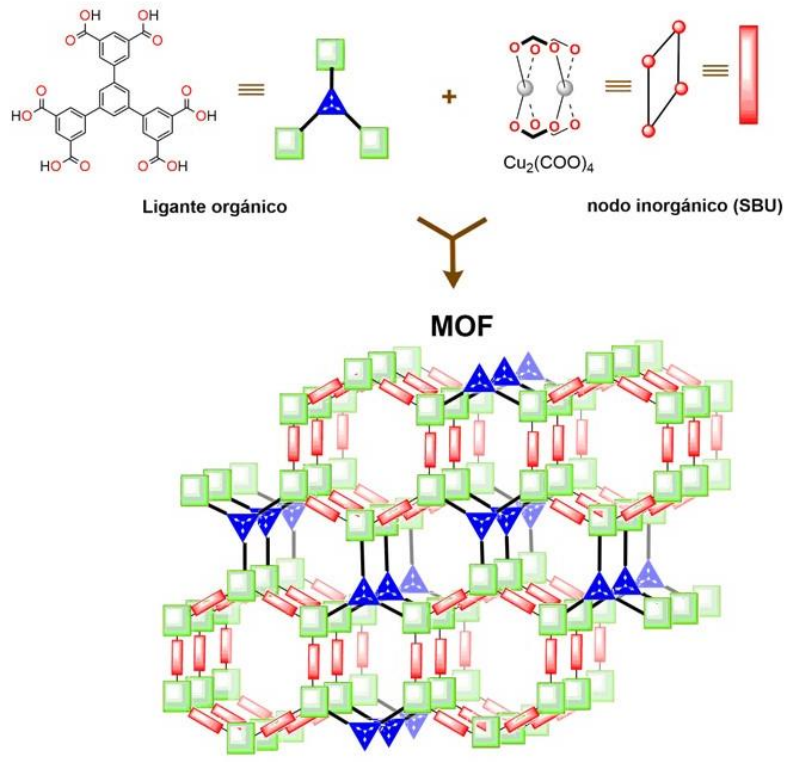

Figura 1: Representación esquemática de los componentes de un MOF. (Cui, 2016)

\section{Historia y antecedentes}

La historia de este tipo de compuestos surge a principios de la década de los 90s, donde la investigación de materiales con estructuras poliméricas, porosas, conteniendo en su estructura iones metálicos y ligantes orgánicos puente aumentó considerablemente, refiriéndose a ellos como: "polímeros de coordinación" los cuales pueden tener estructuras en 1,2 o 3 dimensiones como se ilustra en la Figura 2.

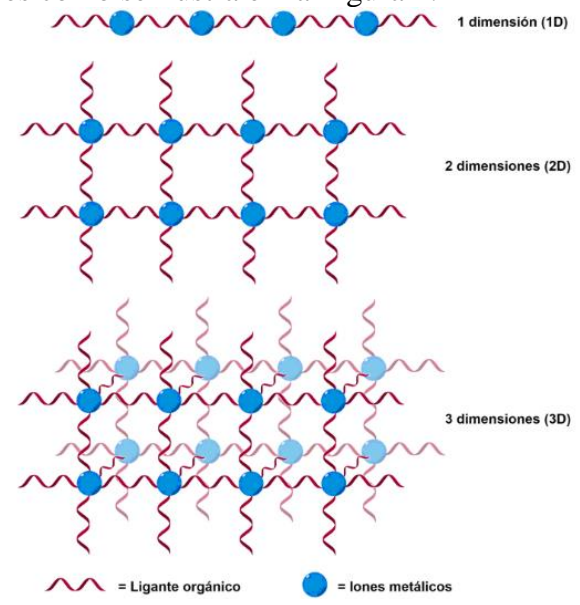

Figura 2: Estructuras esquemáticas de polímeros de coordinación en una (1D), dos (2D) y tres (3D) dimensiones.

En la actualidad el término "estructuras metal-orgánicas" MOF es generalmente utilizado para referirse a las estructuras porosas que presentan un arreglo ordenado entre iones o cúmulos metálicos y ligantes orgánicos formando en su mayoría estructuras cristalinas bi- (2D) o tridimensionales (3D) (Furukawa, 2013). En la Figura 3 se muestra la estructura de un MOF conocido como IRMOF-18 (Rowsell, 2004a) cuya fórmula es $\left[\mathrm{Zn}_{4} \mathrm{O}(\mathrm{L})_{3}\right]$ en donde L es el ligante orgánico 2,3,5,6-tetrametiltereftalato.

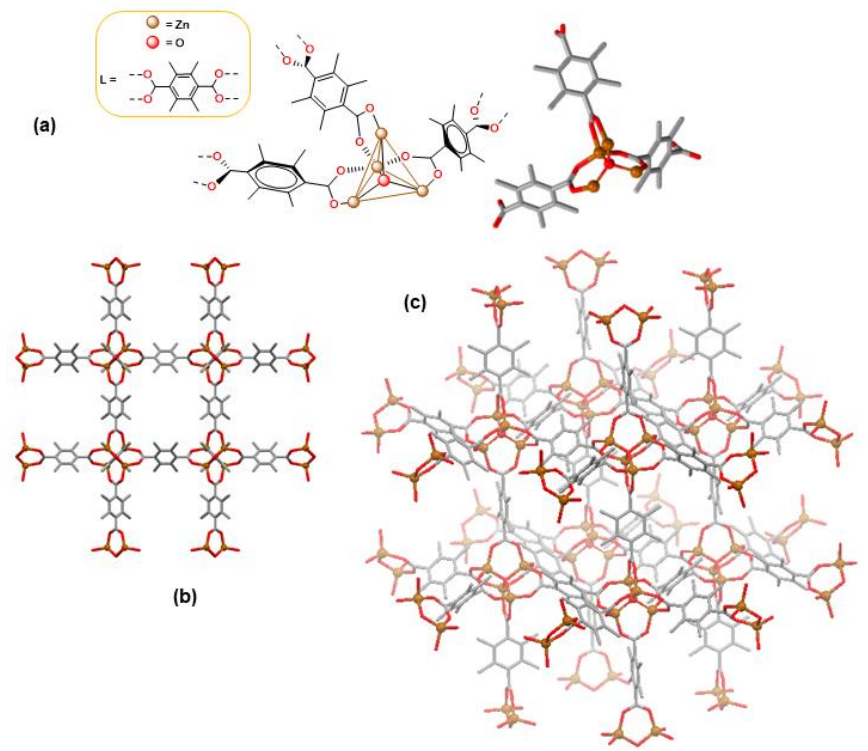

Figura 3: Estructura del IRMOF-18 (Rowsell, 2004a). a) Fórmula y estructura de $\left[\mathrm{Zn}_{4} \mathrm{O}(\mathrm{L})_{3}\right], \mathrm{L}=$

OOC $\left(\mathrm{C}_{6}\left(\mathrm{CH}_{3}\right)_{4}\right) \mathrm{COO}$. b) Estructura en 2D. c) Estructura en

3D. Las estructuras se dibujaron utilizando datos de difracción de rayos $\mathrm{X}$ de monocristal. Los hidrógenos se han omitido por claridad.

Los MOF, al tener arquitecturas tridimensionales, generan cavidades vacías en la red cristalina llamadas poros, que tienen diámetros uniformes, típicamente en el rango de 3 a $20 \AA$, lo que los hace materiales altamente porosos (Ahmad, 2015). En la Figura 4 se muestra la estructura del MOF-5 o IRMOF-1 de fórmula química $\left[\mathrm{Zn}_{4} \mathrm{O}(\mathrm{BDC})_{3}\right], \mathrm{BDC}=1$,4-bencendicarboxilato, en donde se pueden apreciar los poros formados en la estructura cristalina de la especie y cuyo diámetro interno es de $18.5 \AA$ ( $\mathrm{Li}$, 1999).

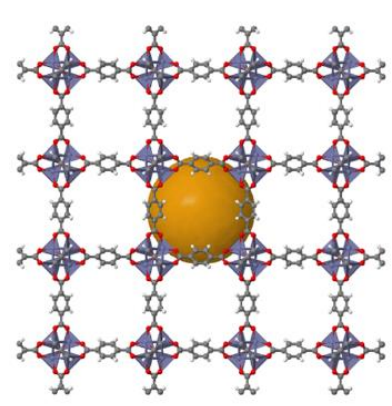

(a)

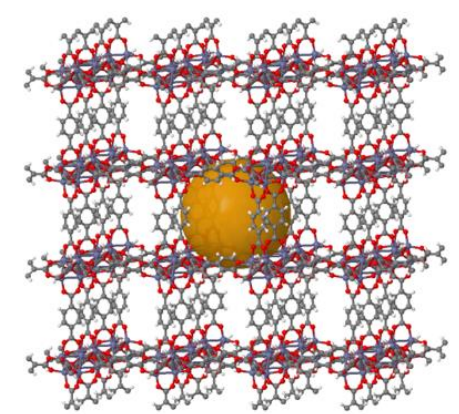

(b)
Figura 4: Estructura del MOF-5. La SBU de $\mathrm{ZnO}_{4}$ tetraédrica esta indica en azul. a) Estructura en 2D. b) Estructura en 3D. La esfera naranja representa el poro interno de $18.5 \AA$ de diámetro. Imagen generada usando el programa ChemTube3D (The University of Liverpool \& Greeves, 2019).

Aunado a la porosidad, otras propiedades físicas relevantes de los MOF son áreas superficiales internas grandes, sin precedente, mayores a $7000 \mathrm{~m}^{2} / \mathrm{g}$ con un límite teórico de área superficial de $14600 \mathrm{~m}^{2} / \mathrm{g}$ o mayores; también tienen volúmenes de espacio 
vacío grandes de entre 55 - 90\% del total de ocupación, y bajas densidades con valores de entre 0.21 a $1.00 \mathrm{~g} / \mathrm{cm}^{3}$, lo que genera estructuras sólidas únicas, con una relación superficie-peso alta y una estabilidad (robustez) grande. Además de propiedades químicas como son un ajuste estructural controlado, procesos de funcionalización convenientes de los ligantes orgánicos y altas estabilidades térmicas y químicas. Por lo que debido a estas propiedades los MOF son especies muy promisorias para el almacenaje de gases, procesos de separación/purificación, detección molecular, administración de fármacos y catálisis heterogénea (Ahmad, 2015).

Los primeros reportes acerca de polímeros de coordinación fueron descritos por Robson, Moore, Yaghi y Zaworotko entre el año 1989 a 1995, (James, 2003). Ellos describieron un procedimiento nuevo para crear estructuras novedosas para el desarrollo de materiales innovadores que ofrecían un cambio radical en sus propiedades. No fue hasta el año de 1995 que Omar Yaghi, separó el término "polímeros de coordinación" de las estructuras metal-orgánicas, definiendo que: para que un sólido se etiquete como una estructura metal-orgánica MOF, debe presentar ciertas propiedades inherentes que implican: a) enlaces que proporcionen robustez al compuesto (es decir, que le confieran estabilidad), b) unidades de enlace que estén disponibles para su modificación por síntesis y c) una estructura geométrica bien definida (Rowsell, 2004b). De esta forma el profesor Yaghi fue considerado como el pionero dentro del campo de la química reticular (como también se le conoce a la química de MOF) abriendo camino hacia la generación de nuevos compuestos de este tipo.

En la Figura 5 se muestra una línea de tiempo que muestra el avance que han tenido los MOF a lo largo de la historia y algunas de las aplicaciones más relevantes que se han identificado (Sharmin, 2016;Li, 1999; Eddaoudi, 2002; Rowsell, 2006; Savonnet, 2011; Imaz, 2010; Briones, 2016; Abney, 2017, Hanikel, 2019; Rozveh, 2020).

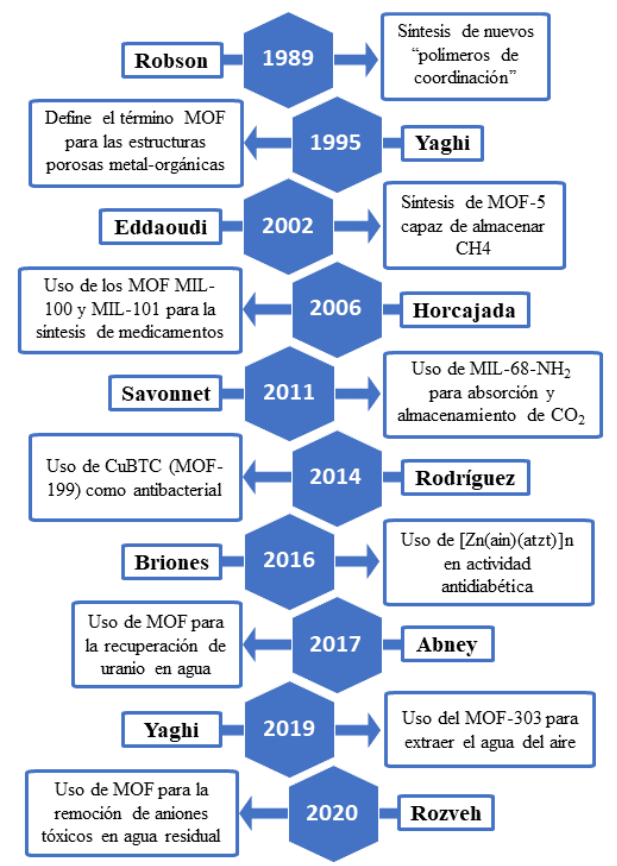

Figura 5: Línea del tiempo en la síntesis de MOF.

Desde esas épocas hasta la actualidad se han sintetizado una gran cantidad de estructuras metal-orgánicas y sus métodos de síntesis han sido muy variados, cabe mencionar que muchas de ellos requieren altas temperaturas y tiempos largos de reacción (ver sección 3.7). Además, se ha observado que muchas veces el cambio de disolvente en donde se lleva a cabo la reacción permite obtener distintos tipos de arreglos espaciales en el estado sólido tanto en 2D como en 3D. Estos arreglos son debidos a diversos factores, como la distribución en el espacio, la conectividad, la porosidad, la reactividad y el tipo de metal con el que se encuentren funcionalizados.

\section{Estructura y métodos de síntesis}

Los compuestos de tipo MOF han sido ampliamente estudiados por la gran versatilidad que han demostrado tener al ser sintetizados, esto es debido a la gran variedad de ligantes orgánicos y iones metálicos (nodos) o mejor conocidos como unidades secundarias de enlace (Secondary Building Units, SBU, por sus siglas en inglés) que pueden ser utilizados para diseñar estas estructuras metal-orgánicas, con una amplia cantidad de arreglos espaciales lo que ha generado una gran variedad de estructuras (Yaghi, 2003).

Debido a que la cantidad y variedad de procedimientos sintéticos y estructuras es muy extensa y va más allá del objetivo de esta revisión, solo se mencionan algunas características generales que se deben considerar para la síntesis de estructuras metal-orgánicas, principalmente en los ligantes (unidades de construcción) comúnmente usados para sintetizar MOF, y que están basados en grupos carboxilatos como puntos de unión.

La mayoría de los MOF se han sintetizado siguiendo principalmente protocolos sintéticos solvotérmicos, ya sea a reflujo $\mathrm{o}$ en condiciones ambientales $\mathrm{y}$, aunque no existen métodos generalizados la síntesis reticular, que es la construcción lógica de redes a partir de bloques de construcción moleculares, ha dado como resultado una gran variedad de estructuras metalorgánicas, algunas de ellas isoreticulares (IR), esto es, que tiene la misma topología subyacente en las estructuras. Es importante resaltar que la adecuada selección del ligante orgánico, de la sal metálica y del disolvente, así como la concentración y la temperatura de reacción son los puntos más relevantes por considerar en la síntesis de MOF; de tal forma que la combinación de la robustez y las estructuras geométricamente bien definidas de las SBU con la selección adecuada de ligantes orgánicos facilitan la síntesis de MOF (Eddaoudi, 2001).

A continuación, se describen algunas características que deben tener los ligantes orgánicos y la forma en que se pueden obtener las SBU de diferentes centros metálicos.

Un de las variables que influyen en la síntesis de estructuras metal-orgánicas son los ligantes orgánicos, que se pueden dividir en tres componentes los cuales se menciona a continuación y algunos ejemplos relacionados se muestran en la Figura 6 (Yaghi, 2019).

- La unidad central

- Las unidades de extensión

- Los grupos de unión

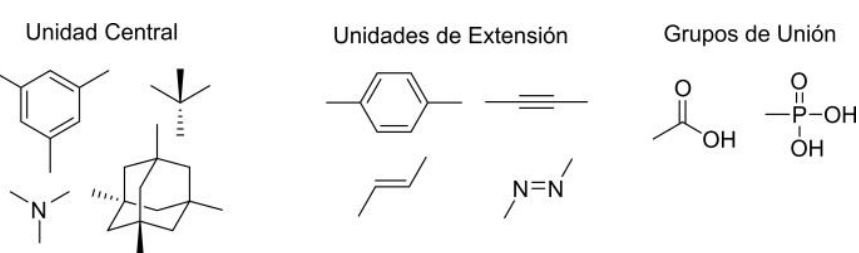

Figura 6: Unidades más comunes en la formación de ligantes orgánicos.

La unidad central se define así, debido a que es la base que permite dar forma y rigidez a la estructura de los ligantes 
orgánicos. Además de ser la parte responsable de la geometría que tome el ligante, los grupos químicos más utilizados para llevar a cabo esta función son: carboxilatos, fosfatos, pirazoles, tetrazoles, catecoles e imidazoles.

Las unidades de extensión son los que permiten modular la distancia y rigidez entre una unidad central y el centro metálico, generando que el tamaño de poro creado se vea afectado. Este tipo de unidades de extensión está formado por interacciones carbóncarbón, carbón-heteroátomo o heteroátomo-heteroátomo.

Los grupos de unión o conectores son los que permiten llevar a cabo la unión del ion o cúmulo metálico con los ligantes orgánicos. Desde mediados del siglo XX se han usado ligantes neutros como bipiridina y nitrilo para formar estos enlaces, pero años después fueron reemplazados por otro tipo de grupos funcionales, como los carboxilatos, ya que la presencia de este tipo de conectores tiene ciertas ventajas sobre los anteriores, como mantener cargas neutras, formación de quelatos (lo que permite tener estructuras más rígidas), formación de cúmulos polinucleares (SBU) y la formación de enlaces fuertes entre los conectores y los iones o cúmulos metálicos (Tranchemontagne, 2009).

Por tal motivo, una parte fundamental de la estructura en los MOF son los ligantes orgánicos, ya que, la geometría de la estructura metal-orgánica se verá afectada dependiendo del número de puntos de unión (conexión) y la cantidad y tipo de enlaces que puedan formar con los iones metálicos de las SBU. Por otro lado, la fuerza de esos enlaces metal-ligante dependen de las características del ion metálico como son su estado de oxidación, valencia, electronegatividad, tamaño, y número de coordinación, por mencionar algunas y, por lo tanto, habrá cambios en sus propiedades físicas como porosidad, área superficial interna y densidad etc., lo que influirá en su potencial aplicación.

Las geometrías que pueden adquirir los MOF pueden ser lineales, trigonales, cuadradas, tetraédricas, etc., y dependen de los puntos de unión o conectores que el ligante orgánico tenga y que se emplean para la síntesis; estos puntos de unión pueden ir desde dos hasta ocho, como se describe a continuación:

\subsection{Dos puntos de unión}

Este tipo de ligantes permiten tener una geometría lineal, angular y en forma desplazada, donde lo único que permite el cambio de estructura es la unidad central. El conector orgánico lineal más común, es el ácido teraftálico o ácido 1,4bencendicarboxilico (BDC) en el cual la unidad central es un anillo aromático y en donde dos grupos carboxilo se encuentran en posición para y funcionan como grupos de unión. En el caso de la forma angular se emplea el ácido isoftálico, que tiene dos grupos carboxilo en posición orto. Para el caso de la geometría desplazada se encuentra el ácido 4,4'-azobenceno dicarboxílico, que debido a la geometría del enlace $\mathrm{N}=\mathrm{N}$, en el centro del compuesto, permite tener una geometría lineal distorsionada del ligante como se muestra en la Figura 7. (Eddaoudi, 2002; Kaskel, 2016).

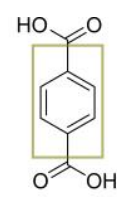

a) b)

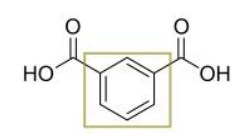

c)

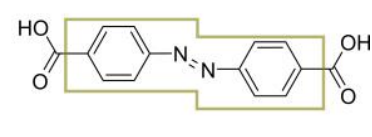

Figura 7: a) Ácido teraftálico, b) Ácido isoftálico y c) Ácido 4,4'-azobencenodicarboxílico.

\subsection{Tres puntos de unión}

Este tipo de ligantes permite generar una geometría trigonal plana alrededor del nodo metálico, pero puede ir variando la simetría del conector dependiendo de la unidad central y las unidades de extensión. Por ejemplo, el ácido trimésico o ácido 1,3,5-bencentricarboxilico (BTC) es un conector altamente simétrico que presenta la geometría de un triángulo equilátero con un grupo puntual de simetría $\mathrm{D}_{3} \mathrm{~h}$. Para obtener ligantes asimétricos se requiere reemplazar el grupo carboxílo por ligantes como el ácido naftoico o el ácido benzoico para obtener compuestos como el ácido 6,6',6"-(benceno-1,3,5-tril)-tris(2naftoico) y el ácido (1,1'-bifenil)-3,4',5-tricarboxilico respectivamente (Chui, 1999; He, 2013; Wang, 2009). En la Figura 8 se muestra la estructura química de los tres ácidos mencionados.

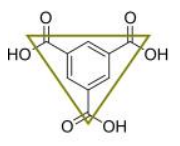

a)

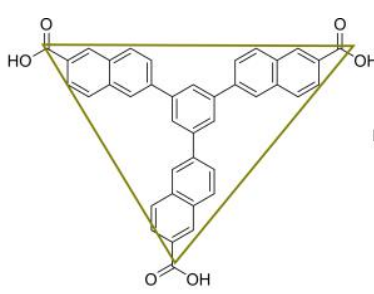

b)

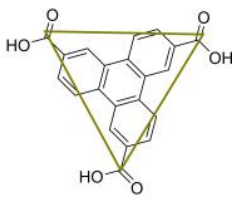

c)
Figura 8: a) Ácido trimésico, b) Ácido 6,6',6"-(benceno-1,3,5tril)-tris(2-naftoico) y c) Ácido (1,1'-bifenil)-3, 4',5tricarboxilico.

\subsection{Cuatro puntos de unión}

Este tipo de ligantes permite la formación de geometrías cuadradas, tetraédricas y algunas otras irregulares como rectangulares o angulares. Para el caso de conectores cuadrados se utilizan las porfirinas, el ejemplo más común es el ácido $\left(4,4^{\prime}, 4^{\prime \prime}, 4^{\prime}\right.$-(porfirin-5,10,15,20-tetrail)tetrabenzoico. Para el caso de estructuras tetraédricas los átomos centrales de carbono o silicio son los más utilizados ya que cuenta con una hibridación $s p^{3}$, como es el caso del ácido 4,4',4",4"'-(adamantano-1,3,5,7tetrail)tetrabenzoico. Para obtener una geometría rectangular se requiere alargar la distancia entre los conectores un ejemplo es el ácido [1,1'-bifenil]-3,3',5,5'-tetracarboxílico, cuyas estructuras se muestran en la Figura 9 (Morris, 2012; Kosal, 2002).

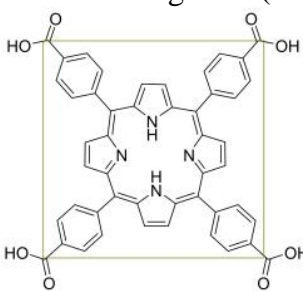

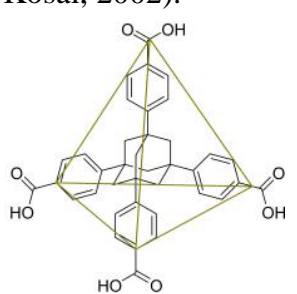

b)

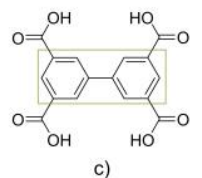

Figura 9: a) Ácido $\left(4,4^{\prime}, 4^{\prime \prime}, 4^{\prime}\right.$-(porfirin-5,10,15,20tetrail)tetrabenzoico, b) Ácido 4,4',4",4"'-(adamantano1,3,5,7-tetrail)tetrabenzoico y c) Ácido [1,1'-bifenil $]-3,3$ ',5,5'tetracarboxílico.

\subsection{Cinco puntos de unión}

Este tipo de conectores son poco usuales debido a la baja simetría que tienen como es el caso del ácido 5'-(4-carboxifenil)[1,1':3,1"-terfenil]-3,3",5,5"-tetracarboxílico, mostrado en la Figura 10 (Schnobrich, 2010). 


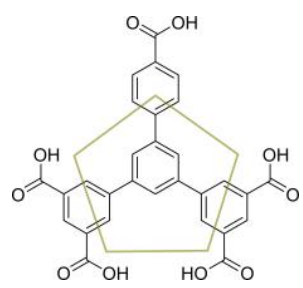

Figura 10: Á ácido 5'-(4-carboxifenil)-[1,1':3,1"-terfenil]$3,3^{\prime \prime}, 5,5^{\prime \prime}$-tetracarboxílico.

\subsection{Seis puntos de unión}

Este tipo de conectores fueron utilizados por primera vez en 2001 (Jia, 2011), en ellos la geometría central es la misma que se emplea cuando se tienen tres puntos de contacto, pero en este caso se reemplaza el ácido benzoico por un ácido isoftálico, un ejemplo es el ácido 5',5",5'"'-(benceno-1,3,5-triil)tris-[1,1':3',1"'-terfenil]4,4"dicarboxílico, mostrado en la Figura 11.

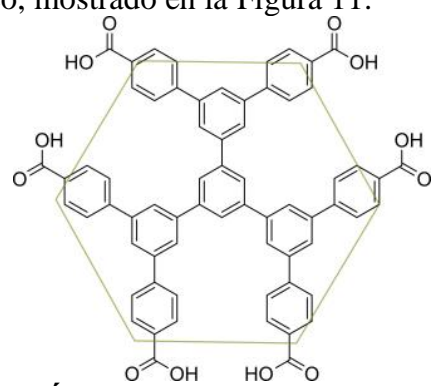

Figura 11: Ácido 5',5",5"'-(benceno-1,3,5-triil)tris$\left[1,1^{\prime}: 3^{\prime}, 1^{\prime \prime}\right.$-terfenil]-4,4"dicarboxílico.

\subsection{Ocho puntos de unión}

Este tipo de ligantes se basa en el grupo central de conectores de 4 puntos de extensión, en donde se difuncionalizan con ácidos carboxílicos siendo uno de los más comunes el ácido 4',4",4"',4"'metanotetrailtetrakis ([1,1'-bifenil]3,5-dicarbixílico) cuya estructura se muestra en la Figura 12 (Tanh Jeazet, 2011).

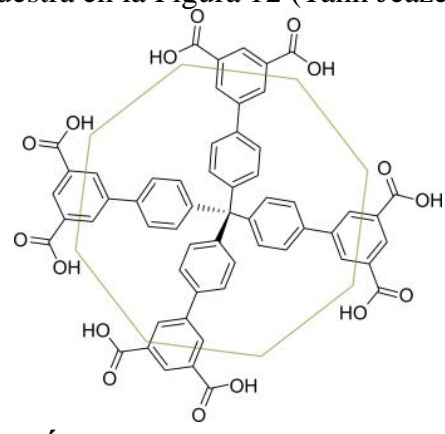

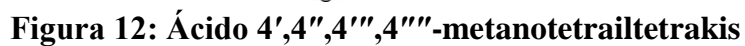
([1,1'-bifenil]3,5-dicarbixílico).

Existen distintas maneras en que se puede unir el centro metálico con el ligante orgánico a través de los grupos de unión, y se les llaman modos de coordinación. Como se observa en la Figura 13 esta unión se puede dar mediante un enlace iónico, de forma monodentada, mediante la formación de quelatos simétricos o asimétricos y mediante la formación de puentes.

Por otro lado, un aspecto muy importante a considerar para la síntesis de MOF son las unidades secundarias de enlace (SBU), que se refiere a los iones o cúmulos metálicos dentro de las estructuras metal-orgánicas, algunas geometrías que presentan estas SBU se muestran en la Figura 14. La alta conectividad que presentan y las diversas estructuras que pueden llegar a formar, los convierte en unidades ideales para una gran variedad de diseños de MOF. (Yaghi, 2003) a)<smiles>[R]C(=O)O[Na]</smiles>

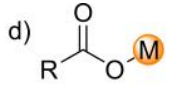

b)<smiles>[M]O[W]=C([R])O[R]</smiles>

c) e) $\overbrace{}^{O}$<smiles>[R]OC([R])=O</smiles>

Figura 13: Formas de unión metal-conector: a) Iónico, b) Quelato (sym), c) Unión (syn-syn), d) Unidentado, e) Quelato (anti) y f) Puente.

Diferentes metales y metaloides de la tabla periódica han sido incluidos en las SBU de las estructuras de los MOF. Por ejemplo, elementos de los bloques $s$ y $p$ empleados incluyen metales de los grupos 1, 2, 13, 14 y 15; mientras que, de los metales de transición, se han empleado a todos los metales de la $1^{\mathrm{a}}$ serie, así como $\mathrm{Y}, \mathrm{Zr}$, $\mathrm{Cd}$, Hf y W, no sin mencionar que se han usado a todos los elementos lantánidos, con excepción del prometio, Pm. (Yaghi, 2019).

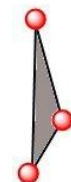

(a)

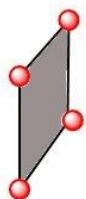

(b)

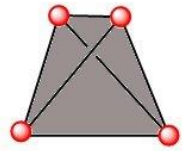

(c)

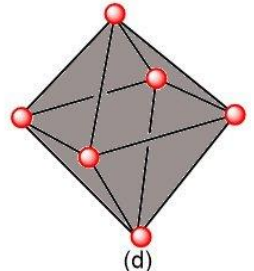

(d)

Figura 14: Estructuras poliédricas de las unidades secundarias de enlace SBU. a) triángulo, b) cuadrado, c) tetraedro, d) octaedro.

Las SBU se forman de manera in situ por lo que su acoplamiento es lento lo que favorece su crecimiento ordenado y por lo tanto la formación de estructuras cristalinas. Para que las SBU se puedan formar es importante tener en cuenta la naturaleza del catión metálico relacionada a su estado de oxidación, valencia y modos de coordinación. Por lo que los métodos de síntesis más comunes se han clasificado con base a la valencia de los metales, que está relacionado con el número de enlaces que el ion metálico puede formar con el ligante orgánico a través de los puntos de unión y que de alguna forma influyen en su coordinación hacia el ligante, aquí se menciona algunas características sintéticas de solo tres tipos de metales.

\subsection{Metales divalentes}

Los metales divalentes como $\mathrm{Ca}^{2+}, \mathrm{Be}^{2+}, \mathrm{Zn}^{2+}$ y $\mathrm{Cu}^{2+}$ tienen un carácter iónico parcial y se han mostrado patrones de energía en donde el enlace metal-carboxilato deja de ser covalente y se convierte en un enlace iónico. La síntesis de MOF de metales divalentes involucra preparar una solución del ligante orgánico a utilizar con disolventes a base de amida, como N,Ndimetilformamida (DMF). Es necesario emplear preferentemente sales metálicas a base de iones nitrato y disolverlas en disolventes apropiados que sean miscibles con el disolvente en donde se encuentra el ligante orgánico. Estas dos soluciones se deben mezclar y calentar en un rango que puede variar desde temperatura ambiente hasta $140^{\circ} \mathrm{C}$. Importante de considerar es que se deben emplear disolventes que ayuden a la solubilidad de los compuestos empleados como materias primas. (Banerjee, 2016).

\subsection{Metales trivalentes}

Para el caso de los metales trivalentes como $\mathrm{In}^{3+}$ los MOF se deben preparar usando disolventes orgánicos como DMF, 
dimetilacetamida (DMA) y N-metil-2-pirolidona (NMP) con temperaturas por debajo de $\operatorname{los} 150^{\circ} \mathrm{C}$, mientras que para $\mathrm{Al}^{3+}$ y $\mathrm{Ga}^{3+}$ se utilizan temperaturas entre los $120-220^{\circ} \mathrm{C}$ en soluciones acuosas básicas. El empleo de ácido nítrico o clorhídrico favorece la formación de enlaces metal-carboxilato en el MOF y la obtención de compuestos con estructuras cristalinas (Riechens, 2005).

Para metales de transición como $\mathrm{Fe}^{3+}, \mathrm{Cr}^{3+}$ y $\mathrm{V}^{3+}$, las condiciones de reacción cambian debido a su alta reactividad además de la capacidad de formar enlaces fuertes metalcarboxilato. Los óxidos e hidróxidos metálicos de estos cationes trivalentes usados para la síntesis se forman en un amplio rango de $\mathrm{pH}$ por lo que se tiene un margen estrecho en las condiciones de reacción para la formación de MOF cristalinos. El control del $\mathrm{pH}$ que permite a una especie de hierro catiónico ser soluble se encuentra por debajo de un valor de $\mathrm{pH}$ de 2, por lo tanto, una síntesis exitosa de MOF de hierro a partir de soluciones neutras o básicas solo se puede lograr mediante la adición de moduladores fuertemente ácidos como ácidos minerales inorgánicos (HF o $\mathrm{HCl})$ o ácidos carboxílicos fuertes $\left(\mathrm{CF}_{3} \mathrm{COOH}\right.$ o $\left.\mathrm{HCOOH}\right)$ y por lo tanto durante la reacción se debe mantener un ambiente ácido con un $\mathrm{pH} \leq 2$; Para $\mathrm{Fe}^{3+}$ se requieren temperaturas menores a $150^{\circ} \mathrm{C}$ mientras que para $\mathrm{Cr}^{3+}$ mayores a $180^{\circ} \mathrm{C}$. Para el caso de $\mathrm{V}^{3+}$ se deben cuidar las condiciones oxidantes ya que puede oxidarse a $\mathrm{V}^{4+}$ (Czaja, 2009).

\subsection{Metales tetravalentes}

Para el caso de metales tetravalentes como $\mathrm{Zr}^{4+}$ y $\mathrm{Hf}^{4+}$ se utilizan las sales $\mathrm{MOCl}_{2}, \mathrm{M}\left(\mathrm{SO}_{4}\right)_{2}$ y $\mathrm{MX}_{4}$, donde $\mathrm{X}=\mathrm{Cl}, \mathrm{Br}$, I y $\mathrm{M}=\mathrm{Zr}^{4+} \mathrm{y} \mathrm{Hf}^{4+}$. Es necesario utilizar una mezcla de disolventes a base de amidas como DMF y ácido fórmico para los ligantes orgánicos y se utilizan temperaturas entre $50-140^{\circ} \mathrm{C}$. Mientras que para $\mathrm{Ti}^{4+}$ se emplean materias primas altamente reactivas como el $\mathrm{Ti}(\mathrm{OR})_{4}$; donde $\mathrm{R}=\mathrm{Me}$ (metilo), Et (etilo), iPr (isopropilo), $\mathrm{Bu}$ (butilo), disolventes orgánicos como DMF y elevadas temperaturas, $100-160^{\circ} \mathrm{C}$, y por tiempos prolongados de reacción 2 a 7 días (Bai, 2016; Nguyen, 2016).

Por otro lado, es importante mencionar que durante la síntesis de los MOF "moléculas huésped" pueden quedar ocluidas en las cavidades de la estructura porosa. Estas "moléculas huésped" pueden ser moléculas del disolvente empleado en la reacción de síntesis o incluso moléculas de las materias primas utilizadas. Por lo que la activación de una estructura metal-orgánica requiere la remoción de estas moléculas huésped de los poros de la estructura, lo cual se puede llevar a cabo mediante varios pasos de lavado e intercambio de disolventes bajo vacío dinámico, logrando con ello la activación de la estructura porosa del MOF. De tal forma que el disolvente de la mezcla de reacción se intercambia por otros disolventes para eliminar los materiales de partida y los subproductos sin reaccionar de los poros del MOF, generalmente son disolventes de punto de ebullición inferior que son apropiados para una activación adicional o posterior, por lo general se usa diclorometano, cloroformo, acetona, etanol o metanol (Yaghi, 2019).

\section{Aplicaciones}

A lo largo de los años el estudio de nuevas estructuras metalorgánicas MOF ha ido en aumento, por tal motivo es importante conocer los avances y aplicaciones que se han tenido tanto en el área de la química como en el desarrollo tecnológico. Los MOF tienen varios campos de aplicación como por ejemplo en almacenamiento y separación de gases, como materiales con propiedades ópticas, conductoras y magnéticas, como sensores químicos, y como catalizadores en catálisis heterogénea y fotocatálisis, y en biomedicina para la administración de fármacos, en la Figura 15 se representan algunas de estas aplicaciones (Li, 2016; Hendon, 2017).

La aplicación de los MOF en estas distintas áreas de la química se debe a sus propiedades químicas y físicas inherentes, propiedades como gran porosidad, áreas superficiales internas y volúmenes de espacio grandes, densidades bajas, relación superficie-peso alta, robustez, y estabilidades térmicas y químicas altas que los ha llevado a tener una gran variedad de estructuras (Ahmad, 2015). A continuación, se describen algunos ejemplos de estos campos de aplicación en donde los MOF se han visto inmersos.

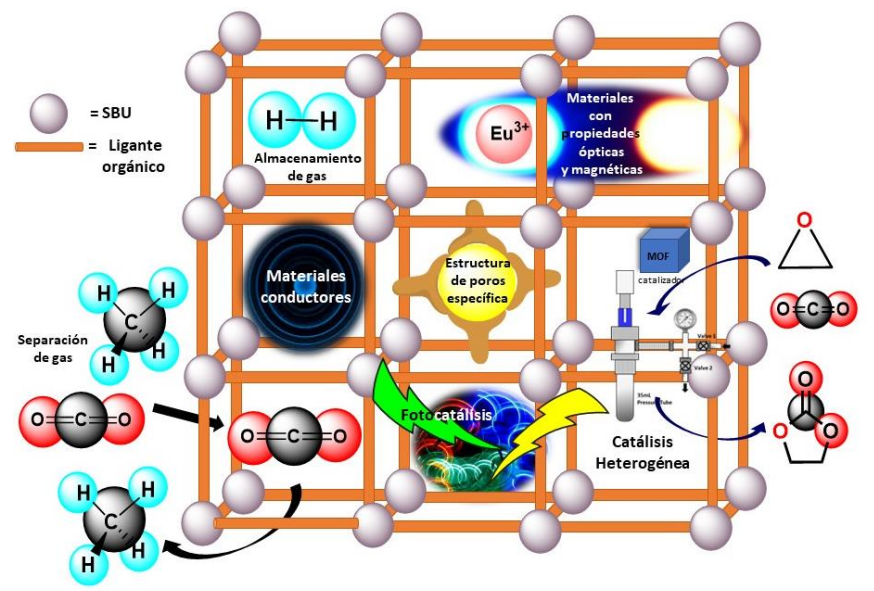

\section{Figura 15: Representación esquemática de diferente aplicaciones de los MOF.}

\subsection{Almacenamiento y separación de gases}

En esta área los MOF han tenido un gran avance, como primer antecedente de este tipo de aplicación se encuentra la síntesis del IRMOF-1 o MOF-5 por el grupo de profesor Yaghi (Li, 1999) y que junto con otros MOF isoreticulares (IRMOF-1, 11, 8 y 18) pueden almacenar hidrógeno $\left(\mathrm{H}_{2}\right)$, y otros gases como metano $\left(\mathrm{CH}_{4}\right)$ en los poros de la estructura. Este subconjunto de materiales fue empleado por su gran área superficial y la sutil diferencia química en sus ligantes, los cuales forman estructuras lineales con dos puntos de unión. Cada estructura se construye uniendo octaédricamente $\mathrm{SBU}$ de $\mathrm{Zn}_{4} \mathrm{O}\left(\mathrm{CO}_{2}\right)$ con seis unidades orgánicas, en la Figura 16 se muestran la estructura de estos IRMOF isoreticulares. Como resultado, los sitios de superficie correspondientes al componente inorgánico (SBU) deben ser idénticos en cada caso, y cualquier diferencia en la capacidad de almacenar hidrógeno puede atribuirse a diferencias en las unidades orgánicas (Rowsell, 2004a).

También se han descrito trabajos de almacenamiento de $\mathrm{CH}_{4}$ y $\mathrm{CO}_{2}$, es así como Long y colaboradores sintetizaron un MOF flexible de $\mathrm{Co}^{2+}$, el [Co(bdp)]; bdp=1,4-bencendipirazolato que es un ligante lineal con dos puntos de unión. Este MOF-Co es capaz de almacenar a $25^{\circ} \mathrm{C}, 155 \mathrm{~cm}^{3}$ de metano a 35 bar de presión y hasta $197 \mathrm{~cm}^{3}$ de metano a 65 bar de presión (Mason, 2015).

Otro ejemplo es el MOF-210 que tiene la capacidad de almacenar hasta $2870 \mathrm{mg} / \mathrm{g}$ de $\mathrm{CO}_{2}$ a temperatura ambiente a 50 bar de presión. La estructura de este MOF está formada por SBU de $\mathrm{Zn}^{2+}$ y ligantes orgánicos lineales el bifenildicarboxilato (BPDC) y trigonales el 4,4',4"-(bencen-1,3,5-triiltris(etino-2,1diil))tribenzoato (BTE) con dos y tes puntos de unión respectivamente, lo que permite maximizar su superficie y el tamaño de poro (Rowsell, 2006; Yaghi, 2011). 


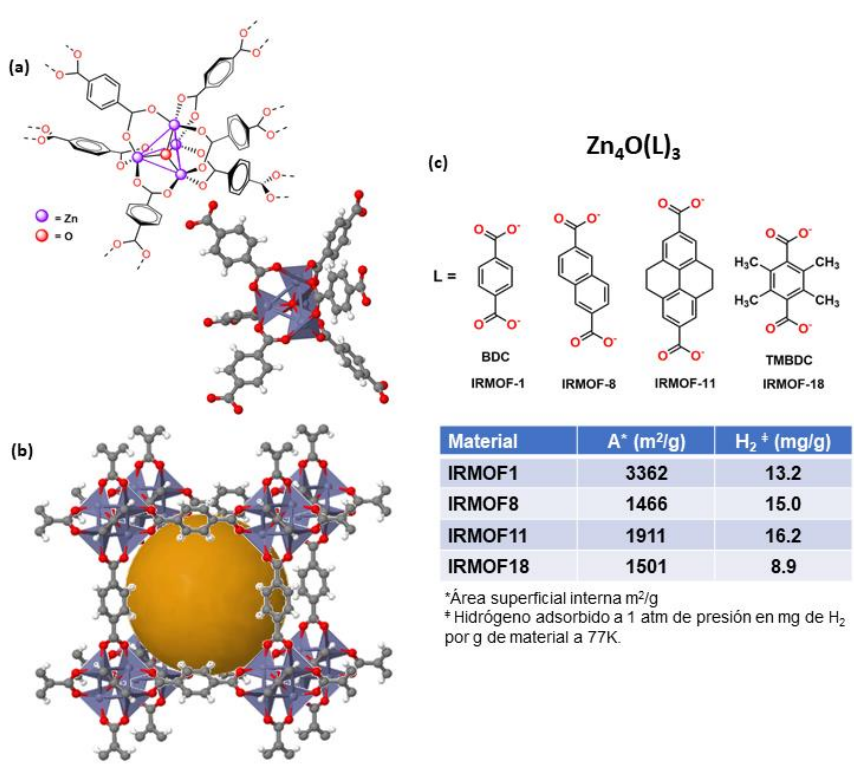

Figura 16: MOF isoreticulares $\mathrm{Zn}_{4} \mathrm{O}(\mathrm{L})_{3}$ constituidos por SBU de cúmulos de óxido de zinc y ligantes lineales carboxilato L. a) Unidad octaédrica $\mathrm{SBU}$ de $\mathrm{Zn}_{4} \mathrm{O}\left(\mathrm{CO}_{2}\right)$ con seis unidades orgánicas. b) La esfera naranja representa la cavidad (poro) del IRMOF-5. Imagen generada usando el programa ChemTube3D (The University of Liverpool \&

Greeves, 2019). c) Ligantes orgánicos lineales con dos puntos de unión y datos de área superficial y absorción de $\mathbf{H}_{2}$ (Rowsell, 2004a).

Con respecto a la separación de gases, las estructuras metalorgánicas se han utilizado para separar hidrocarburos ligeros, como es el caso del Fe-MOF-74 el cual permite la obtención y separación de compuestos con una pureza del 99 al $99.5 \%$, el MgMOF-74 es capaz de absorber un $37.9 \%$ de $\mathrm{CO}_{2}$ (Bloch, 2012). El ZIF-8 presenta cierta selectividad frente a otros gases como lo son $\mathrm{O}_{2} / \mathrm{N}_{2}, \mathrm{H}_{2} / \mathrm{N}_{2}, \mathrm{H}_{2} / \mathrm{CH}_{4}$ debido a su estructura de tipo zeolita con ligantes imidazolatos, lo que genera cambios en el volumen de espacio libre, en su permeabilidad, adsorción, difusión y en el tamizado molecular (Bushell, 2013, Bux, 2011).

Recientemente se reportó la síntesis del MOF-303 de $\mathrm{Al}^{3+}$ de fórmula $[\mathrm{Al}(\mathrm{OH})(\mathrm{HPDC})] ; \quad \mathrm{HPDC}=1 \mathrm{H}$-pirazol-3,5dicarboxilato, que es un ligante lineal con dos puntos de unión que tiene la capacidad de filtrar agua (Fathieh, 2018; Hanikel, 2019). El MOF-303 absorbe las moléculas de agua que se encuentran en el aire, para posteriormente liberarlas mediante cambios de temperatura, lo que ha dado como resultado la obtención de agua altamente pura lista para consumo humano, como se esquematiza en la Figura 17. Esta estructura metal-orgánica es capaz de filtrar hasta $1.3 \mathrm{~L}$ por $1 \mathrm{~kg}$ de MOF-303.

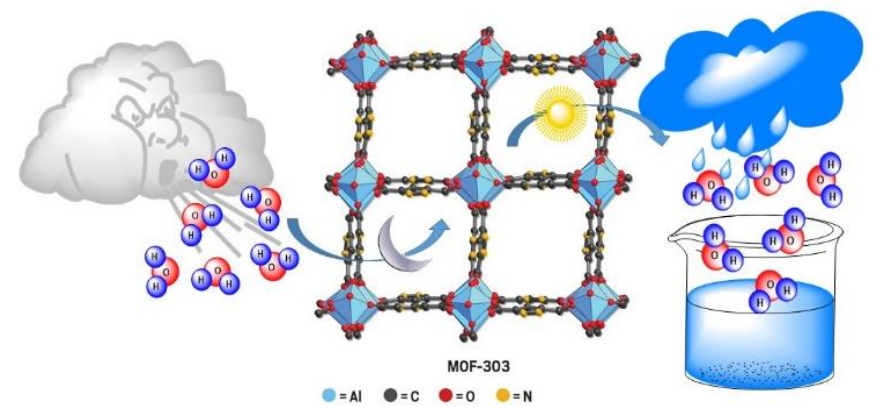

Figura 17: MOF-303 llamado "cosechador de agua". Fuente: “MOF-303" by Nikita Hanikel/Yaghi research group. (Hanikel, 2019).

\subsection{Materiales ópticos}

Un MOF altamente poroso de cadmio $\left(\mathrm{Cd}^{2+}\right)$ y un complejo de iridio $\left(\mathrm{Ir}^{3+}\right)$ se han empleado para la fabricación de diodos emisores de luz (lighth emmitting diode, LED, por sus siglas en inglés) con longitudes de onda diferentes. Esta nueva estrategia de síntesis y técnica de elaboración de los diodos consistió en encapsular al complejo catiónico de iridio, $\left[\left(\operatorname{Ir}(\mathrm{ppy})_{2}(\mathrm{bpy})\right]^{+},(\mathrm{ppy}\right.$ $=2$-fenilpiridina, bpy $=2,2^{\prime}$-bipiridina) en un MOF aniónico de cadmio, $\left[\left(\mathrm{CH}_{3}\right)_{2} \mathrm{NH}_{2}\right]_{15}\left[\left(\mathrm{Cd}_{2} \mathrm{Cl}\right)_{3}(\mathrm{TATPT})_{4}\right]$, $($ TATPT $=2,4,6-$ tris(2,5-dicarboxil fenilamino)-1,3,5-triazina), y que presenta una estructura tridimensional con dos cavidades de 2 y $3 \mathrm{~nm}$ de tamaño de poro. Al irradiar con luz ultravioleta a $365 \mathrm{~nm}$, el MOF aniónico y el MOF con el complejo de Iridio (MOF-Ir) a dos concentraciones distintas, a una concentración de $3.5 \%$ en peso y a una concentración de $8.8 \%$ en peso, resultó en emisiones de luz de color azul, blanca y amarilla respectivamente, lo cual abre nuevas perspectivas de aplicación de los MOF en el campo de la luminiscencia. (Sun, 2013).

Chen y colaboradores sintetizaron un MOF-Ln (en donde Ln es un ion lantánido), el $\left[\mathrm{Er}_{2}\left(\mathrm{BDC}-\mathrm{F}_{4}\right)_{3}(\mathrm{DMF})\right]$ que contiene ligantes orgánicos fluorados el BDC-F4 = 2,3,5,6-tetrafluoro-1,4bencendicarboxilato o tetrafluorotereftalato. Los átomos de flúor mejoran significativamente la intensidad luminiscente del ion $\mathrm{Er}$ al reducir el efecto de la fluorescencia debida al enlace $\mathrm{C}-\mathrm{H}$ que se observa en el ligante análogo sin átomos de flúor, el BDC = 1,4-bencendicarboxilato, en una relación $3: 1$. Y por lo tanto se mejoran sus propiedades de luminiscencia en el infrarrojo cercano (Near Infrared, NIR, por sus siglas en inglés), mediante la absorción de cationes como $\mathrm{Er}^{3+}(1530 \mathrm{~nm}), \mathrm{Nd}^{3+}(1060 \mathrm{~nm})$ y $\mathrm{Yb}^{3+}$ (980nm) (Chen, 2006). Este tipo de experimentos tienen aplicación en el campo militar, para producir aparatos de visión nocturna, para análisis espectroscópicos, etc.

Asimismo, se ha descrito la fabricación de un microlaser a través del uso de un MOF aniónico de $\mathrm{Zn}^{2+}$ (bio-MOF-1), el $\left[\mathrm{Zn}_{8}(\mathrm{Ad})_{4}(\mathrm{BPDC})_{6} \mathrm{O}\right], \quad(\mathrm{Ad}=$ adeninato; $\mathrm{BPDC}=$ bifenildicarboxilato), el cual en soluciones orgánicas es capaz de encapsular colorantes catiónicos. Este tipo de tecnología es utilizada para el bombardeo de fotones, el almacenamiento de datos y en terapia fotodinámica (Yu, 2013).

\subsection{Materiales conductores}

En el ámbito de los conductores eléctricos se busca el transporte eficiente de electrones, por lo que una forma efectiva de promover la deslocalización de electrones en los MOF es a través de la deslocalización electrónica a lo largo de sus enlaces, mediante la resonancia de electrones de tipo $\pi$ como ocurre en los enlaces de compuestos aromáticos (Nagapradeep, 2018). El MOF $\mathrm{Cu}\left[\mathrm{Cu}(\mathrm{pdt})_{2}\right]$ (pdt $=2,3$-pirazintiolato), contiene dos tipos de centros metálicos de cobre con geometría cuadrada plana y es capaz de conducir hasta $6 \times 10^{-4} \mathrm{~S} / \mathrm{cm}$ (unidades de conducción electrolítica) comportándose como un semiconductor, con una activación energética de $193 \mathrm{mV}$ a temperatura ambiente (Takaishi, 2009). Este tipo de compuestos podrían ser usados como para la fabricación de baterías, pilas de combustible, condensadores, etc.

En 2012, Gándara y colaboradores (Gándara, 2012) demostraron los beneficios de introducir iones de $\mathrm{Fe}^{2+}$ en la síntesis de materiales conductores, observando que un MOF de $\left[\mathrm{Fe}(1,2,3 \text {-triazol })_{2}\right]$ es capaz de conducir hasta $7.7 \times 10^{-5} \mathrm{~S} / \mathrm{cm}$ a temperatura ambiente. Posteriormente también se introdujeron elementos como $\mathrm{Mg}^{2+}, \mathrm{Mn}^{2+}, \mathrm{Co}^{2+}, \mathrm{Cu}^{2+}$, and $\mathrm{Zn}^{2+}$ utilizando como ligante orgánico el 1,2,3-triazol para la formación de MOF. 


\subsection{Materiales magnéticos}

Los materiales magnéticos moleculares han demostrado tener cierto interés debido a su potencial aplicación en almacenamiento de información de gran densidad y en dispositivos electrónicos a nanoescala, los MOF debido a la incorporación de metales paramagnéticos o ligantes orgánicos paramagnéticos, que cuentan con electrones desapareados, dan la posibilidad de crear materiales magnéticos ( $\mathrm{Li}, 2016)$.

En este caso la naturaleza porosa de los MOF se ha aprovechado para encapsular diversas moléculas magnéticas, tal es el caso del MOF-Ln ( $\mathrm{Ln}=\mathrm{Tb}$, Dy, Ho, Er), estudios de susceptibilidad magnética a diferentes temperaturas mostraron que la estructura metal-orgánica que contiene disprosio (Dy) presenta una relajación más lenta bajo un campo magnético de $1000 \mathrm{Oe}$, lo que sugiere que las interacciones ferromagnéticas dominan. Existiendo una transferencia intramolecular de energía más lenta que va desde del ligante al ion de Dy, este proceso es conocido como sensibilización luminiscente y provoca que el estado triplete asociado al proceso de fluorescencia sea más prolongado y por lo tanto tenga una relajación más lenta. Esto implica que el estado excitado toma más tiempo en regresar a su estado basal, teniendo como resultado propiedades fluorescentes y un comportamiento ferromagnético más grandes, lo cual fue sustentado por dinámicas de magnetización a diferentes frecuencias concluyendo que este compuesto podría ser usado por su propiedades como magneto molecular único (Single Molecule Magnet, SMM, por sus siglas en inglés) (Xu, 2013).

\subsection{Sensores químicos}

La aplicación de los MOF como sensores químicos es posible debido a la relación huésped-anfitrión que se puede dar dentro de los MOF, la estructura cristalina de estas especies permite determinar, la presencia de sitios metálicos abiertos (Open Metal Sites, OMS, por sus siglas en inglés) referido a sitios metálicos coordinativamente insaturados o sitios que se comporten como ácido-base de Lewis, esto es, que tengan pares de electrones libres que inicien la coordinación específica hacia los centros metálicos, que actúan como ácidos de Lewis, o una interacción enlazante hacia átomos de hidrógeno u otras especies deficientes de electrones y lograr de esta forma una mayor sensibilidad en el reconocimiento molecular (Li, 2016; Chen, 2010; Hu, 2017).

Un ejemplo de esto es el MOF $\left[\mathrm{Zn}_{2}(\mathrm{BPDC})_{2}(\text { bpee })_{2}\right]$ cuya estructura se muestra en la Figura 18, que es un material luminiscente y microporoso, que permite el almacenamiento traza de explosivos como el 2,4-dinitrobenceno, por lo que es utilizado como sensor en la detección de explosivos. (Roales, 2017; Lin, 2016).

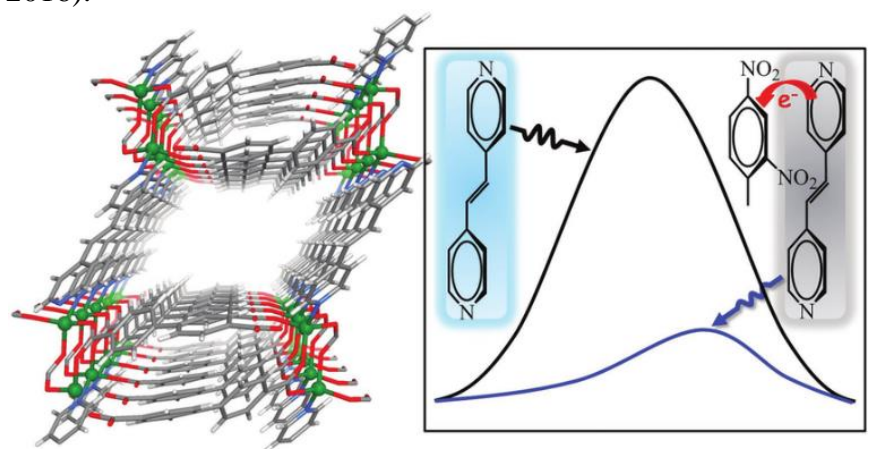

Figura 18: Estructura 3D del MOF [ $\left.\mathbf{Z n}_{2}(\text { BPDC })_{2}(\text { bpee })_{2}\right]$. Fuente: (Lin, 2016), License: Creative Commons Attribution 4.0 International.
MOF en películas delgadas depositadas solvotérmicamente sobre diferentes soportes se han diseñado. Existen varios métodos para fabricar películas de MOF uno de ellos es el depósito de películas de MOF directamente sobre sustratos desnudos o sustratos funcionalizados con moléculas orgánicas, entre los sustratos más comunes está el uso de oro, obleas de silicio, grafeno, vidrio, óxido de indio y estaño (Indium Tin Oxide, ITO, por sus siglas en inglés), óxido de aluminio poroso y recientemente se reportó el uso de partillas de $\mathrm{Gd}_{2} \mathrm{O}_{3}$ (Liu, 2016). Siendo el ITO uno de los soportes más usados cuyas propiedades como baja resistividad del orden de $10^{-4} \Omega \mathrm{cm}$ y alta transparencia del orden del $85 \%$, son adecuadas para usarlo como soporte transparente conductor (Quiñones Segura, 2013; Demichelis, 1985). A la fecha, algunos MOF luminiscentes en película se han diseñado como sensores de oxígeno, los cuales presentan excelentes propiedades mecánicas y alta estabilidad química incluso en una atmósfera ácida (Liu, 2014), por lo que se considera que tienen aplicaciones potenciales en ciencias de los alimentos, análisis ambiental, investigación espacial y bioquímica.

Por ejemplo, Dou y colaboradores (Dou, 2014) reportaron la síntesis de dos MOF luminiscentes el CMP-5 y el MIL-100 de $\mathrm{In}^{3+}$, en donde se usó un ligante orgánicos con tres puntos de unión el 1,3,5-bencentricarboxilato, y que mediante una posterior funcionalización encapsularon $\mathrm{Tb}^{3+}$. Con ellos se hicieron películas de MOF luminiscente preparadas in situ por el método de síntesis solvotérmica usando ITO como soporte. Las películas tuvieron un grosor de $2.5 \mu \mathrm{m}$, determinado por microscopia electrónica de barrido, presentando una alta porosidad y una rápida y reversible detección de oxígeno molecular, por lo que este tipo de compuestos tiene un gran potencial de aplicación como sensores de gases o de vapores por su alta sensibilidad $\left(\mathrm{K}_{\mathrm{sv}}=7.59\right.$ $\operatorname{bar}^{-1}$ ). Los valores de sensibilidad para la detección de oxígeno están basados en la medición de la constante de Stern-Volmer $\left(\mathrm{K}_{\mathrm{sv}}\right)(\mathrm{Liu}, 2014)$, y se ha descrito que pueden ir desde 1 hasta 356 $\mathrm{bar}^{-1}$ (Lin, 2016), lo cual indica un intervalo que puede de bajaalta-extrema sensibilidad.

\subsection{Catálisis heterogénea}

En esta área se llevan a cabo procesos en diferentes fases en donde el catalizador es sólido y las especies a catalizar están en solución y debido a que los MOF son sólidos insolubles o poco solubles en disolventes orgánicos es por lo que se han empleado en la catálisis heterogénea. Así los iones metálicos como el $\mathrm{Cu}^{2+}$, $\mathrm{Zn}^{2+}$ y algunos otros iones lantánidos, como $\mathrm{La}, \mathrm{Ce}, \mathrm{Pr}, \mathrm{Nd}, \mathrm{Sm}$, $\mathrm{Eu}, \mathrm{Gd}, \mathrm{Tb}, \mathrm{Dy}, \mathrm{Ho}, \mathrm{Er}, \mathrm{Yb}$ (Gándara, 2008), dentro de las estructuras MOF tienen cierta afinidad por moléculas de disolvente orgánico, las cuales pueden quedar incrustadas en los poros de la estructura, pero que pueden ser fácilmente removibles para tener vacantes de coordinación, que pueden ser usadas como los sitios activos en una catálisis heterogénea.

Un caso relevante es la estructura metal-orgánica de $\left[\mathrm{Cu}_{2}(\mathrm{Cu}-\right.$ tactmb $\left.)\left(\mathrm{H}_{2} \mathrm{O}\right)_{3}\left(\mathrm{NO}_{3}\right)_{2}\right]$, en donde usaron un ligante orgánico macrocíclico el ácido 1,4,7,10-tetrazazciclododecano$\mathrm{N}, \mathrm{N}^{\prime}, \mathrm{N}^{\prime \prime}, \mathrm{N}^{\prime \prime \prime}$-tetra- $p$-metilbenzoico (tactmb) que es un ligante con ocho puntos de unión, lo que generó un MOF con una estructura 3D cubo-octaédrica. Este MOF-Cu presentó una actividad catalítica del $95.4 \%$ para la cicloadición de $\mathrm{CO}_{2}$ de epóxidos a temperatura ambiente y con 1 atm de presión (Gao, 2014).

Asimismo, se ha descrito la síntesis de MOF en donde el ácido (S)-3-hidroxi-2(piridin-4-ilmetilamino) ha sido utilizado como ligante orgánico en su formación y que han sido capaces de catalizar la adición de agentes de Grignard a cetonas $\alpha, \beta$ insaturadas para generar alcoholes alílicos terciarios quirales, obteniendo excesos enatioméricos (ee) del 99\%. (Wang, 2009). 


\subsection{Materiales fotocatalíticos}

Para poder realizar fotocatálisis es necesario tener dos componentes básicos característicos que son el fotosensibilizador y el catalizador, por sus propiedades físicas y químicas, los MOF pueden tener ambos componentes o actuar como cualquiera de ellos. Estudios recientes han demostrado que la reducción fotocatalítica de $\mathrm{CO}_{2}$ ha sido muy eficiente en presencia de estructuras metal-orgánicas. La reducción fotocatalítica de $\mathrm{CO}_{2}$ es un proceso químico importante en donde se lleva a cabo la transformación de $\mathrm{CO}_{2}$ (un compuesto contaminante y de efecto invernadero) a compuestos que puede tener un mayor valor agregado (Li, 2016).

Un ejemplo es el MOF [ $\left.\mathrm{NH}_{2}-\mathrm{MIL}-125(\mathrm{Ti})\right]$ el cual pudo transformar $\mathrm{CO}_{2}$ en ácido fórmico $(\mathrm{HCCOH})$ usando acetonitrilo como disolvente y en presencia de trietanolamina (TEOA) bajo irradiación de luz visible, empleando una lámpara de Xe con un rango de luz de 420-800 nm. La presencia del anión $\mathrm{HCCO}^{-}$fue detectada mediante cromatografía de iones en fase líquida. Los resultado obtenidos mostraron un cambio en la concentración de $\mathrm{HCOO}^{-}$con respecto al tiempo de irradiación obteniéndose un máximo de $8.14 \mu \mathrm{mol}$ en $10 \mathrm{~h}$ y que la ausencia de luz no genera al ácido fórmico, por lo que la reacción es fotocatalítica. Este MOF tiene una fórmula general $\left[\mathrm{Ti}_{8} \mathrm{O}_{8}(\mathrm{OH})_{4}\left(\text { bdc- } \mathrm{NH}_{2}\right)_{6}\right]$, bdc$\mathrm{NH}_{2}=$ 2-amino-bencen-1,4-dicarboxilato, en donde el cúmulo metálico $\mathrm{Ti}_{8} \mathrm{O}_{8}(\mathrm{OH})_{4}$ está rodeado de seis ligantes orgánicos derivado de ácido 2-aminotereftálico. Se confirmó que la actividad fotocatalítica de la luz visible para la reducción de $\mathrm{CO}_{2}$ es inducida por la funcionalidad amino $\left(-\mathrm{NH}_{2}\right)$, ya que en condiciones similares análogo MOF [MIL-125(Ti)] derivado del ácido bencen-1,4-dicarboxilico no tuvo actividad. Los autores proponen que en el mecanismo de reducción del $\mathrm{CO}_{2}$ inicialmente ocurre una absorción de luz en la banda de transferencia de carga del ligante bdc- $\mathrm{NH}_{2}$ al centro metálico en el MOF, lo que genera la transferencia de un electrón al $\mathrm{Ti}^{4+}$ reduciéndolo a $\mathrm{Ti}^{3+}$, la TEOA actúa como donador de electrones y cuando se introduce $\mathrm{CO}_{2}$ se lleva a cabo su reducción a $\mathrm{HCOO}^{-}$por parte del $\mathrm{Ti}^{3+}$, lo que regenera el ciclo fotocatalítico al obtener nuevamente $\mathrm{Ti}^{4+}$. (Fu, 2012).

\subsection{MOF a nanoescala para biomedicina}

Los MOF presentan una gran área superficial con poros de tamaño nanométrico como una característica única, lo cual ha permitido el almacenamiento, entrega y control de medicamentos (Li, 2016).

El grupo del profesor Férey fue de los primeros en explorar la utilidad potencial de los MOF porosos para el suministro controlado de drogas (Horcajada, 2006). Ellos lograron encapsular ibuprofeno en un $60 \%$ en dos compuestos MOF de $\mathrm{Cr}^{3+}$, el MIL-100 y el MIL-101 (Férey, 2005), estos compuestos presentaron un mejor porcentaje de encapsulamiento en comparación con otros materiales mesoporosos descritos como las estructuras orgánicas covalentes (Covalent Organic Frameworks, COF, por sus siglas en inglés) específicamente los PI-COF-4 and PI-COF-5 (Fang, 2015) o los MOF flexibles MIL-53(Cr) y MIL$53(\mathrm{Fe})$ que solo encapsularon el $20 \%$ de ibuprofeno (Horcajada, 2008).

Horcajada estudio la cinética del suministro de ibuprofeno a fluido corporal simulado (Simulated Body Fluid, SBF, por sus siglas en inglés) (Yilmaz, 2020) a $37^{\circ} \mathrm{C}$, con agitación continua en donde la concentración de ibuprofeno administrado se determinó por cromatografía de líquidos (High-Performance Liquid Chromatography, HPLC; por sus siglas en inglés). Estos MOF contienen diferentes ligantes orgánicos el $\mathrm{BTC}=1,3,5$ bencentricarboxilato que es un ligante trigonal con tres puntos de unión y el $\mathrm{BDC}=1$,4-bencendicarboxilato que (como ya se mencionó antes) es un ligante lineal con dos puntos de unión, respectivamente y se describe que la adsorción de ibuprofeno es resultado de sus diferentes tamaño de poro. El MIL-100 tiene una cavidad más pequeña de 25 a 29 Å comparada con la del MIL-101 de 29 a $34 \AA$ y a pesar de que el MIL-101 tiene volúmenes de poro mayores (entre 12700 y $20600 \AA^{3}$ ) que el MIL-100 (8200 y $12700 \AA^{3}$ ), el MIL-100 adsorbe $0.35 \mathrm{~g}$ de ibuprofeno por gramo de material mientras que el MIL-101 mostró una adsorción del medicamento de 1.4 gramos de ibuprofeno por gramo de material, algo sin precedentes, siendo esto una ventaja ya que solo se requieren cantidades muy pequeñas de material para la administración de dosis altas. Las razones estructurales pueden explicar esta discrepancia, particularmente las dimensiones de las ventanas de las redes en relación con las de ibuprofeno, con lo que se demuestra que el control del tamaño de poro en los MOF es de gran importancia para su aplicación (Horcajada, 2006).

En la liberación del ibuprofeno o ácido isobutilfenil propiónico $\left(\mathrm{C}_{13} \mathrm{H}_{18} \mathrm{O}_{2}\right)$ del MIL-100 y MIL-101 se pueden tener diferentes etapas relacionadas con la ubicación del medicamento dentro de las estructuras. Para cada tipo de estructura dos situaciones pueden ocurrir con el huésped (ibuprofeno): 1) cuando el huésped están cerca de las paredes del MOF la interacción huésped-anfitrión está regida por interacciones electrostáticas anión-catión e interacciones tipo $\pi-\pi$ entre los anillos aromáticos del ibuprofeno y la parte orgánica del MOF y dependen del tamaño del poro; 2) llenos los poros las moléculas de ibuprofeno restantes ocupan el espacio de salida de las cavidades, con solo interacciones de ibuprofeno-ibuprofeno. La liberación inicial de ibuprofeno, dentro de las primeras dos horas, está referida solo a las moléculas débilmente unidas, la liberación completa de ibuprofeno se logra después de tres días de ensayos in vitro para el MIL-100 y toma seis días con el MIL-101, lo que puede deberse a la mayor proporción de anillos aromáticos en el compuesto que aumenta el número de interacciones entre el ibuprofeno y la superficie de los poros (Horcajada, 2006).

También se ha probado el almacenamiento y liberación de otros medicamentos antiarritmias como la procainamida (An, 2009) y la cafeína (Cunha, 2013) o anticancerígenos como el 5-Fu (Sun , 2011).

Recientemente Lin y colaboradores (Deng, 2015) describieron que la estructura MOF basada en materiales de carboxilato de hierro (III) [MIL-100 (Fe)] posee alta porosidad y no es toxico, como el cromo, lo que es de gran valor como nanoportadores para el almacenamiento y suministro de fármacos, así como para terapia de bioimagen dirigidas en tratamientos anticancerígenos. Las nanopartículas formadas por este MOF dopado con iones lantánidos $\mathrm{Yb}^{3+} / \mathrm{Eu}^{3+}$ es una nanoplataforma única como un vehículo híbrido inorgánico-orgánico para el suministro de medicamentos y bioimagen dirigidos en el diagnóstico y terapia temprana de células cancerosas, debido a sus propiedades ópticas únicas que proporcionan un fondo de autofluorescencia mínimo, una profundidad de penetración de luz profunda y un daño mínimo a los organismos vivos para la obtención de imágenes ópticas. Este tipo de nanoacarreadores pueden moléculas pequeñas como ácido fólico, péptidos o aptámeros en su sistema de administración de fármacos dirigido debido a que presentan una unión altamente específica con células cancerosas. En este estudio se utilizó el aptámero anticancerígeno AS1411, un oligonucleótido, con resultados prometedores en el aumento de la eficiencia quimioterapéutica y el monitoreo de la progresión del cáncer simultáneamente.

\section{Conclusiones}


El uso exitoso de unidades de construcción secundarias (SBU) que incluye la selección del catión metálico $\left(\right.$ como $\mathrm{Zn}^{2+}, \mathrm{Cu}^{2+}, \mathrm{Fe}^{3+}$ sólo por mencionar algunos) y de los ligantes orgánicos (unidades de extensión y puntos de unión o conectores) impacta en la formación de las estructuras metal-orgánicas 3D y en sus propiedades físicas, para poder tener una aplicación en un campo específico como se ha visto en los ejemplos mencionados en las aplicaciones.

Es evidente que los investigadores se encuentran en una etapa en la que se puede diseñar la columna vertebral primaria de las estructuras de los MOF con fines específicos. Por ejemplo, la capacidad de diseñar las estructuras de los MOF ha permitido sintetizar compuestos con una estructura de poro específica, esto es controlar el tamaño, forma y función, (IZA Commission on Metal Organic Frameworks, 2013) y por lo tanto ha conseguido aspectos de porosidad diferentes de lo que se ha logrado con otro tipo de materiales porosos conocidos como las zeolitas (IZA Structure commission, 2002).

Las estructuras metal-orgánicas al ser materiales porosos cristalinos formados por iones metálicos y ligantes orgánicos, han demostrado tener un gran número potencial de aplicaciones, como son la creación de nuevos materiales con propiedades fisicoquímicas importantes, para la síntesis de sensores químicos, la formación de diodos emisores de luz, la creación de compuestos fluorescentes y fosforescentes en diferentes regiones del espectro electromagnético alrededor de la región visible, la síntesis de compuestos adsorbentes con áreas superficiales muy grandes con aplicaciones en catálisis, recuperación de iones en tratamiento de aguas residuales, o la obtención de agua a partir del aire, los también llamados cosechadores de agua, entre muchas otras aplicaciones, y que pueden en futuro cercano dar paso a la creación de dispositivos de alta tecnología con aplicaciones en equipos y enseres de uso diario en nuestras vidas, como computadoras, teléfonos celulares, pantallas de TV, equipos científicos de aplicación específica, como microláseres o aparatos de visión nocturna, por mencionar algunos.

Es así que como se describió a lo largo de esta revisión general, aún existe un largo camino por recorrer en el área de los MOF, debido a la versatilidad que muestran para ser sintetizados y la gran variedad de aplicaciones que pudieran tener, por lo que resultan ser compuestos muy interesantes y cada vez más estudiados.

\section{Agradecimientos}

MHVF y MCG agradecen a CONACYT por las becas otorgadas para sus estudios de maestría y doctorado respectivamente.

A la Universidad Autónoma del Estado de Hidalgo por las facilidades otorgadas.

\section{Referencias}

Abney, C. W., Mayes, R. T., Saito, T., Dai, S. (2017). Materials for the Recovery of Uranium from Seawater. Chemical Reviews 117(23), 1393514013. https://doi.org/10.1021/acs.chemrev.7b00355

Ahmad, N., Younus, H. A., Chughtaiab, A. H., Verpoort, F. (2015). MetalOrganic Molecular Cages: Applications of Biochemical Implications. $\begin{array}{llll}\text { Chemical Society } & \text { Reviews } & \text { 94, }\end{array}$ https://doi.org/10.1039/C4CS00222A

An, J., Geib, S. J., Rosi, N. L. (2009). Cation-Triggered Drug Release from a Porous Zinc-Adeninate Metal-Organic Framework. Journal of the American Chemical Society 131(24), 8376-8377. https://doi.org/10.1021/ja902972w
Bai, Y., Dou, Y., Xie, L. (2016). Zr-based Metal-Organic Frameworks: design, Synthesis, Structure and Applications. Chemical Society Reviews 45, 2327-2367. https://doi.org/10.1039/C5CS00837A

Banerjee, D., Wang, H., Deibert, B., Li, J. (2016). Alkaline Earth Metal-Based Metal-Organic Frameworks: Synthesis. The Chemistry of Metal-Organic Frameworks, Properties and applications. In: Kaskel S. (Ed.), The Chemistry of Metal-Organic Frameworks: Synthesis, Characterization, and Applications. Vol. I. Weinheim, Alemania: Wiley-VCH Verlag $\mathrm{GmbH} \&$ Co. Alemania, Ch. 4, pp. 73-103. https://doi.org/10.1002/9783527693078.ch4

Bloch, E. D., Queen, W. L., Krishna, R., Zadrozny, J. M., Brown, C. M., Long, J. R. (2012). Hydrocarbon Separations in a Metal-Organic Framework with Open Iron(II) Coordination Sites. Science 335, 1606-1610. https://doi.org/10.1126/science.1217544

Briones, D., Fernández, B., Calahorro, A. J., Fairen-Jimenez, D., Sanz, R., Martínez, F., Orcajo, G., Sebastián, E. S., Seco, J. M., González, C. S., Llopis, J., Rodríguez-Diéguez, A. (2016). Highly Active Anti-Diabetic Metal-Organic Framework. Crystal Growth \& Design 16, 537-540. https://doi.org/10.1021/acs.cgd.5b01274

Bushell, A. F., Attfield, M. P., Mason, Ch. R., Budd, P. M., Yampolskii, Y., Starannikova, L., ... Isaeva, V. (2013). Gas Permeation Parameters of Mixed Matrix Membranes Based on the Polymer of Intrinsic Microporosity PIM-1 and the Zeolitic Imidazolate Framework ZIF-8. Journal of Membrane Science 427, 48-62. https://doi.org/10.1016/j.memsci.2012.09.035

Bux, H., Chmelik, Ch., Krishna, R., Caro, J. (2011). Ethene/Ethane Separation by the MOF Membrane ZIF-8: Molecular Correlation of Permeation, Adsorption, Diffusion. Journal of Membrane Science 369(1-2), 284-289. https://doi.org/ 10.1016/j.memsci.2010.12.001

Chen, B., Xiang, S., Qian, G. (2010). Metal-Organic Frameworks with Functional Pores for Recognition of Small Molecules. Accounts of Chemical Research 43(8), 1115-1124. https://doi.org/10.1021/ar100023y

Chen, B., Yang, Y., Zapata, F., Qian, G., Luo, Y., Zhang, J., Lobkovsky, E. (2006). Enhanced Near-Infrared-Luminescence in an Erbium Tetrafluoroterephthalate Framework. Inorganic Chemistry 45(22) 8882 8886. https://doi.org/10.1021/ic060568u

Chui, S., Lo, S., Charmant, J., Orpen, A., Williams, I. (1999). A Chemically Functionalizable Nanoporous Material $\left[\mathrm{Cu}_{3}(\mathrm{TMA})_{2}\left(\mathrm{H}_{2} \mathrm{O}\right)_{n}\right.$. Science 283, 1148-1150. https://doi.org/10.1126/science.283.5405.1148

Cui, Y., Li. B., He, H., Zhou, W., Chen, B., Qian, G. (2016). Metal-Organic Frameworks as Platforms for Functional Materials. Account Chemical Research 49, 483-493. https://doi.org/10.1021/acs.accounts.5b00530

Cunha, D., Ben Yahia, M., Hall, Sh., Miller, S. R., Chevreau, H., Elkaïm, E., ... Serre, Ch. (2013). Rationale of Drug Encapsulation and Release from Biocompatible Porous Metal-Organic Frameworks. Chemistry of Materials, 25(14), 2767-2776. https://doi.org/10.1021/cm400798p

Czaja, A. Trukhan, N. Müller, U. (2009). Industrial Applications of MetalOrganic Frameworks. Chemical Society Reviews 38, 1284-1293. https://doi.org/10.1039/B804680H

Demichelis, F., Minetti-Mezzetti, E., Smurro, V., Tagliaferro, A., Tresso, E. (1985). Physical Properties of Chemically Sprayed Tin Oxide and Indium Tin Oxide Transparent Conductive Films. Journal of Physics D: Applied Physics, 18(9), 1825-1832. https://doi.org/10.1088/0022-3727/18/9/013

Deng, K., Hou, Z., Li, X., Li, CH., Zhang, Y., Deng, X., Cheng, Z., Lin, J. (2015). Aptamer-Mediated Up-conversion Core/MOF Shell Nanocomposites for Targeted Drug Delivery and Cell Imaging. Scientific Reports 5:7851. https://doi.org/10.1038/srep07851

Dou, Z., Yu, J., Yang, Y., Wang, Z., Yang, D., Qian, G. (2014). Luminescent Metal-Organic Framework Films as Highly Sensitive and Fast-response Oxygen Sensors. Journal of the American Chemical Society 136(15) 55275530. https://doi.org/10.1021/ja411224j

Eddaoudi, M., Kim, J., Rosi, N., Vodak, D., Wachter, J., O’Keefe, M., Yaghi, O. M. (2002). Systematic Design of Pore Size and Functionality in Isoreticular MOFs and their Application in Methane Storage. Science 295, 469-472. https://doi.org/10.1126/science.1067208

Eddaoudi, M., Moler, D. B., Li, H, Chen, B., Reineke, T. M., O'Keeffe, M. Yaghi O. M. (2001). Modular Chemistry: Secondary Building Units as a Basis for the Design of Highly Porous and Robust Metal-Organic Carboxylate Frameworks. Account Chemical Research 34(4), 319-330. https://doi.org/10.1021/ar000034b

Fang, Q., Wang, J., Gu, Sh., Kaspar, R. B., Zhuang, Zh., Zheng, J., ... Yan, Y. (2015). 3D Porous Crystalline Polyimide Covalent Organic Frameworks for Drug Delivery. Journal of the American Chemical Society 137(26), 8352-8355. https://doi.org/10.1021/jacs.5b04147

Fathieh, F., Kalmutzki, M. J., Kapustin, E. A., Waller, P. J. Yang, J., Yaghi, O. M. (2018). Practical Water Production from Desert Air. Science Advances 4, 6, eaat3198. https://doi.org/10.1126/sciadv.aat3198 
Férey, G., Mellot-Draznieks, C., Serre, C., Millange, F., Dutour, J., Surble, S., Margiolaki, I. (2005). A Chromium Terphthalate-Based Solid with Unusually Large Pore Volumes and Surface Area. Science 309, 20402042. https://doi.org/10.1126/science.1116275

Fu, Y., Sun, D., Chen, Y., Huang, R., Ding, Z., Fu, X., Li, Z. (2012). An Amine Functionalized Titanium Metal-Organic Framework Photocatalyst With Visible-Light-Induced Activity for $\mathrm{CO}_{2}$ Reduction. Angewandte Chemie International Edition 51, 3364-3367. https://doi.org/10.1002/anie.201108357

Furukawa, H., Cordova, K. E., O’Keeffe, M., Yaghi, O. M. (2013). The Chemistry and Applications of Metal-Organic Frameworks. Science 341(6149), 1230444. https://doi.org/10.1126/science.1230444

Gándara, F., Andrés, A. de, Gómez-Lor, B., Gutiérrez-Puebla, E., Iglesias, M., Monge, M. A., ... Snejko, N. (2008). A Rare-Earth MOF Series: Fascinating Structure, Efficient Light Emitters, and Promising Catalysts. Crystal Growth \& Design 8(2), 378-380. https://doi.org/10.1021/cg700796m

Gándara, F., Uribe-Romo, F. J., Britt, D. K., Furukawa, H., Lei, L., Cheng, R., Duan, X., O'Keeffe, M., Yaghi, O. M. (2012). Porous, Conductive MetalTriazolates and Their Structural Elucidation by the Charge-Flipping Method. Chemistry European Journal 18, 10595-10601. https://doi.org/10.1002/chem.201103433

Gao, W. Y., Chen, Y., Niu, Y., Williams, K., Cash, L., Perez, P. J., Wojtas, L., Cai, J., Chen, Y. S., Ma, S. (2014). Crystal Engineering of an NBO Topology Metal-Organic Framework for Chemical Fixation of $\mathrm{CO}_{2}$ under Ambient Conditions. Angewandte Chemie 126, 2653-2657. https://doi.org/10.1002/anie.201309778

Hanikel, N., Prévot, M. S., Fathieh, F., Kapustin, E. A., Lyu, H., Wang, H., Diercks, N. J., Glover, T. G., Yaghi, O. M. (2019). Rapid Cycling and Exceptional Yield in a Metal-Organic Framework Water Harvester. ACS Central Science 5, 1699-1706. https://doi.org/10.1021/acscentsci.9b00745

He, Y., Guo, Z., Xiang, S., Zhang, Z., Zhou, W., Fronczek, F., Parkin, S., Hyde, S., O'Keeffe, M., Chen, B. (2013). Metastable Interwoven Mesoporous Metal-Organic Frameworks. Inorganic Chemistry 52(19), 11580-11584. https://doi.org/10.1021/ic401870e

Hendon, C., Rieth, A., Korzýnski, M., Dincâ, M. (2017). Grand Challenges and Future Opportunities for Metal-Organic Frameworks. ACS Central Science 3(6), 554-563. https://doi.org/10.1021/acscentsci.7b00197

Horcajada, P., Serre, C., Maurin, G., Ramsahye, N. A., Balas, F., Vallet-Regí, M., ... Férey, G. (2008). Flexible Porous Metal-Organic Frameworks for a Controlled Drug Delivery. Journal of the American Chemical Society, 130(21), 6774-6780. https://doi.org/10.1021/ja710973k

Horcajada, P., Serre, Ch., Vallet-Regí, M., Sebban, M., Taulelle, F., Férey, G. (2006). Metal-Organic Frameworks as Efficient Materials for Drug Delivery. Angewandte Chemie International Edition 45(36), 5974-5978. https://doi.org/10.1002/anie.200601878

Hu, Z., Zhao, D. (2017). Metal-Organic Frameworks with Lewis Acidity: Synthesis, Characterization, and Catalytic Applications. CrystEngComm 19(29), 4066-4081. https://doi.org/10.1039/c6ce02660e

Imaz, I, Rubio-Martınez, M, García-Fernandez, L, Garcia, F, Ruiz-Molina, D, Hernando, J, Puntes, V, Maspoch, D. (2010). Coordination Polymer Particles as Potential Drug Delivery Systems. Chemical Communications 46, 4737-4739. https://doi.org/10.1039/C003084H

IZA Commission on Metal Organic Frameworks. (2013, 29 octubre). Recuperado 9 de julio de 2020, de http://www.izaonline.org//MOF/default.htm

IZA Structure commission. (2002, 10 enero). Recuperado 9 de julio de 2020, de http://www.iza-structure.org/

James, S. L. (2003). Metal-Organic Frameworks. Chemical Society Reviews 32, 276-288. https://doi.org/10.1039/B200393G

Jia, J., Sun, F., Fang, Q., Liang, X., Cai, K., Bian, Z., Zhao, H., Gao, L., Zhu, G. (2011). Porous Metal-Organic Frameworks as Platforms for Functional Applications. Chemistry Communications 47, 9167-9169. https://doi.org/10.1039/c1cc12891d

Kaskel, S., Furukawa, H., Sun, X. (2016). Functional Linkers. In: Kaskel S. (Ed.), The Chemistry of Metal-Organic Frameworks: Synthesis, Characterization, and Applications. Vol. I. Weinheim, Alemania: WileyVCH Verlag GmbH \& Co. Alemania, Part II. https://doi.org/10.1002/anie.201611669

Kosal, M., Chou, J., Wilson, S., Suslick, K. (2002). A Functional Zeolite Analogue Assembled from Metalloporphyrins. Nature Materials 1, 118121. https://doi.org/10.1038/nmat730

Li, H., Eddaoudi, M., O'Keeffe, M., Yaghi, O. M. (1999). Design and Synthesis of an Exceptionally Stable and Highly Porous Metal-Organic Framework. Nature 402, 276-279. https://doi.org/10.1038/46248
Li, B., Wen, H., Cui, Y., Zhou, W., Qian, G., Chen, B. (2016). Emerging Multifunctional Metal-Organic Framework Materials. Advanced Materials 28(40), 1-42. https://doi.org/10.1002/adma.201601133

Lin, R.-B., Liu S.-Y., Ye J.-W., Li X.-Y., Zhang, J.-Peng. (2016). Photoluminescent Metal-Organic Frameworks for Gas Sensing. Advance Science 3. n/a-n/a. https://doi.org/10.1002/advs.201500434

Liu, S.-Y., Qi, X.-L., Lin, R.-B., Cheng, X.-N., Liao, P.-Q., Zhang, J.-P., Chen, X.-M. (2014). Porous Cu(I) Triazolate Framework and Derived Hybrid Membrane with Exceptionally High Sensing Efficiency for Gaseous Oxygen. Advanced Functional Materials 24(37), 5866-5872. https://doi.org/10.1002/adfm.201401125

Liu, X., Fu, W., Bouwman, E. (2016). One-Step Growth of Lanthanoid MetalOrganic Framework (MOF) Films under Solvothermal Conditions for Temperature Sensing. Chemical Communications, 52(42), 6926-6929. https://doi.org/10.1039/c6cc01407k

Mason, J. A., Oktawiec, J., Taylor, M. K., Hudson, M. R., Rodriguez, J., Bachman, J. E., Gonzalez, M. I., Cervellino, A., Guagliardi, A., Brown, C. M., Llewellyn, P. L., Masciocchi, N., Long, J. R. (2015). Methane Storage in Flexible Metal-Organic Frameworks with Intrinsic Thermal Management. Nature 527, 357-361. https://doi.org/10.1038/nature15732

Morris, W., Volosskiy, B., Demir, S., Gándara, F., McGrier, P. L., Furukawa, H., ... Yaghi, O. M. (2012). Synthesis, Structure, and Metalation of Two New Highly Porous Zirconium Metal-Organic Frameworks. Inorganic Chemistry 51(12), 6443-6445. https://doi.org/10.1021/ic300825s

Nagapradeep, N., Krishnendu, M., Sourav, S. (2018). Electrically Conductive Metal-Organic Frameworks. In: Shengqian, M., Perman, J. A. (Ed.), Elaboration and Applications of Metal-Organic Frameworks. Vol. I. Word Scientific Pubishing. USA, Ch.15, pp. 655-684. https://doi.org/10.1142/9789813226739_0015

Nguyen, H., Gándara, F., Furukawa, H. (2016). A Titanium-Organic Framework as an Exemplar of Combining the Chemistry of Metal and Covalent-Organic Frameworks. Journal of the American Chemical Society 138(13), 4330-4333. https://doi.org/10.1021/jacs.6b01233

O'keeffe, M., Peskov, M. A., Ramsden, S. J., Yaghi, O. M. (2018). The Reticular Chemistry Structure Resource (RCSR) Database of, and Symbols for, Crystal Nets. Account Chemical Research 41, 1782-1789. https://doi.org/10.1021/ar800124u

Quiñones Segura, C. A. (2013). Síntesis de Películas Transparentes y Conductoras utilizadas en Celdas Solares Sensibilizadas por Colorantes. ELEMENTOS 2(2), 2248-5252. https://doi.org/10.15765/e.v2i2.182

Richens, D. (2005). Ligand substitution reactions at inorganic centers Chemical Reviews 105(6) 1961-2002. https://doi.org/10.1021/cr030705u

Roales, J., Moscoso, F. G., Gámez, F., Lopes-Costa, T., Sousaraei, A., Casado, S., Castro-Smirnov, J. R., Cabanillas-González, J., Almeida, J., Queirós, C., Cunha-Silva, L., Silva A. M. G., Pedrosa, J. M, (2017). Preparation of Luminescent Metal-Organic Framework Films by Soft-Imprinting for 2,4$\begin{array}{lll}\text { Dinitrotoluene } & \text { Sensing. } & \text { Materials }\end{array}$ https://doi.org/10.3390/ma10090992

Rowsell, J. L. C, Millward, A. R., Park, K. S., Yaghi, O. M. (2004a). Hydrogen Sorption in Functionalized Metal-Organic Frameworks. Journal of the American Chemical Society 126(18), 5666-5667. https://doi.org/10.1021/ja049408c

Rowsell, J. L. C., Yaghi, O. M. (2004b). Metal-Organic Frameworks: A New Class of Porous Materials. Microporous and Mesoporous Materials 73(12), 3-14. https://doi.org/10.1016/j.micromeso.2004.03.034

Rowsell, J. L. C., Yaghi, O. M., (2006). Effects of Functionalization, Catenation, and Variation of the Metal Oxide and Organic Linking Units on the Low-Pressure Hydrogen Adsorption Properties of Metal-Organic Frameworks. Journal of the American Chemical Society 128(4), 1304 1315. https://doi.org/10.1021/ja056639q

Rozveh, Z. S., Kazemi, S., Karimi, M., Ali, G. A. M., \& Safarifard, V. (2020). Effect of functionalization of metal-organic frameworks on anion sensing Polyhedron 183, 114514. https://doi.org/10.1016/j.poly.2020.114514

Savonnet, M., Farrusseng, D., Pinel, C., Bazer-Bachi, D., Bats, N., Lecocq, V. (2011). WO 201 1/048284 Al. Lyon, Francia: Demande Internationale Publiee en Vertu du Traite de Cooperation en Matiere de Brevets (PCT).

Schnobrich, J., Lebel, O., Cychosz, K., (2010). Linker-Directed Vertex Desymmetrization for the Production of Coordination Polymers with High Porosity. Journal of the American Chemical Society 132(39), 1394113948. https://doi.org/10.1021/ja107423k

Sharmin, E., Zafar, F. (2016). Introductory Chapter: Metal Organic Frameworks (MOFs) | IntechOpen, DOI: 10.5772/64797. Recuperado 6 de junio de 2020, de https://www.intechopen.com/books/metal-organicframeworks/introductory-chapter-metal-organic-frameworks-mofs-

Sun, Ch.-Y., Qin, Ch., Wang, Ch.-G., Su, Zh.-M., Wang, Sh., Wang, X.-L., .. Wang, E.-B. (2011). Chiral Nanoporous Metal-Organic Frameworks with 
High Porosity as Materials for Drug Delivery. Advanced Materials, 23(47), 5629-5632. https://doi.org/10.1002/adma.201102538

Sun, Ch.-Y., Wang, X.-L., Zhang, X., Qin, Ch., Li, P., Su, Zh.-M., ... Li, J. (2013). Efficient and Tunable White-Light Emission of Metal-Organic Frameworks by Iridium-Complex Encapsulation. Nature Communications 4(1), 1-8. https://doi.org/10.1038/ncomms3717

Takaishi, S., Hosoda, M., Kajiwara, T., Miyasaka, H., Yamashita, M., Nakanishi, Y., ... Kitagawa, H. (2009). Electroconductive Porous Coordination Polymer $\mathrm{Cu}\left[\mathrm{Cu}(\mathrm{pdt})_{2}\right]$ Composed of Donor and Acceptor Building Units. Inorganic Chemistry 48(19), 9048-9050. https://doi.org/10.1021/ic802117q

Tan, C., Yang, S., Champness, N., Lin, X., Blake, A., Lewis, W., Schröder, M. (2011). High Capacity Gas Storage by a 4,8-Connected Metal-Organic Polyhedral Framework. Chemistry Communications 47, 4487-4489. https://doi.org/10.1039/C1CC10378D

The University of Liverpool \& Greeves, N. (2019). MOF-5 (or IRMOF-1) Metal Organic Framework. Recuperado 5 de junio de 2020, de https://www.chemtube3d.com/mof-mof5/

Tranchemontagne, D. J., Mendoza-Cortés, J. L., O’Keeffe, M., Yaghi, O. M. (2009). Secondary Building Units, Nets and Bonding in the Chemistry of Metal-Organic Frameworks. Chemical Society Reviews 38, 1257-1283. https://doi.org/10.1039/B817735J

Xu, W., Zhou, Y., Huang, D., Xiong, W., Su, M., Wang, K., ... Hong, M. (2013). Crystal Structure, Multiplex Photoluminescence, and Magnetic Properties of a Series of Lanthanide Coordination Polymers Based on Quinoline Carboxylate Ligand. Crystal Growth \& Design 13, 5420-5432. https://doi.org/10.1021/cg401391b
Wang, X., Ma, S., Yuan, D., Yoon, J., Hwang, Y., Chang, J., Wang, X. Jørgensen, M., Chen, Y., Zhou, H. (2009). A Large-Surface-Area BoraciteNetwork-Topology Porous MOF Constructed from a Conjugated Ligand Exhibiting a High Hydrogen Uptake Capacity. Inorganic Chemistry 48(16), 7519-7521. https://doi.org/10.1021/ic901073w

Wang, M., Xie, M.-H., Wu, C.-D., Wang, Y.-G. (2009). From One to Three: a Serine Derivate Manipulated Homochiral Metal-Organic Framework. Chemical Communications (17) 2396-2398. https://doi.org/10.1039/b823323c

Yaghi, O. M., O’Keeffe, M., Ockwig, N. W., Chae, H. K. Eddaoudi, M., Kim, J. (2003). Reticular Synthesis and the Design of New Materials. Nature 423, 705-714. https://doi.org/10.1038/nature01650

Yaghi, O. M., Kalmutzki, M., Diercks, C., (2019). Building Units of MOFs. Introduction to Reticular Chemistry. Weinheim, Alemania: Wiley-VCH Verlag GmbH \& Co. Alemania. https://doi.org/10.1002/anie.201906230

Yaghi, O. M. (2011). IV.C.9 Hydrogen Storage in Metal-Organic Frameworks. Recuperado 6 de junio de 2020, de https://www.hydrogen.energy.gov/pdfs/progress11/iv_c_9_yaghi_2011.p df.

Yilmaz, B., Pazarceviren, A. E., Tezcaner, A., Evis, Z. (2020). Historical Development of Simulated Body Fluids Used in Biomedical Applications A review. Microchemical Journal, 155, 104713. https://doi.org/10.1016/j.microc.2020.104713

Yu, J., Cui, Y., Xu, H., Yang, Y., Wang, Z., Chen, B., Qian, G. (2013). Confinement of Pyridinium Hemicyanine Dye within an Anionic MetalOrganic Framework for Two-Photon-Pumped Lasing. Nature communications 4:2719. https://doi.org/10.1038/ncomms3719 